\title{
Correction to: T1 and T2 MR fingerprinting measurements of prostate cancer and prostatitis correlate with deep learning-derived estimates of epithelium, lumen, and stromal composition on corresponding whole mount histopathology
}

\begin{abstract}
Rakesh Shiradkar $^{1}$ (D) - Ananya Panda ${ }^{2,3} \cdot$ Patrick Leo $^{1} \cdot$ Andrew JanowCzyk $^{1} \cdot$ Xavier Farre $^{4} \cdot$ Nafiseh Janaki $^{5,6} \cdot$ Lin Li $^{1}$. Shivani Pahwa ${ }^{3} \cdot$ Amr Mahran $^{7}$. Christina Buzzy ${ }^{7}$. Pingfu Fu ${ }^{8} \cdot$ Robin Elliott $^{5} \cdot$ Gregory MacLennan $^{5} \cdot$ Lee Ponsky $^{7}$. Vikas Gulani $^{3,9} \cdot$ Anant Madabhushi $^{1}$
\end{abstract}

Published online: 18 September 2020

(C) European Society of Radiology 2020

Correction to: European Radiology

https://doi.org/10.1007/s00330-020-07214-9

The original version of this article, published on 02 September 2020, unfortunately contained a mistake. The following correction has therefore been made in the original: The spelling of Pingfu Fu's name was incorrect. The corrected author list is given above. The original article has been corrected.

Publisher's note Springer Nature remains neutral with regard to jurisdictional claims in published maps and institutional affiliations.

The online version of the original article can be found at https://doi.org/ 10.1007/s00330-020-07214-9

Rakesh Shiradkar

rxs558@case.edu

1 Department of Biomedical Engineering, Case Western Reserve University, Cleveland, OH 44106, USA

2 Department of Radiology, Mayo Clinic, Rochester, MN, USA

3 Department of Radiology, University Hospitals Cleveland Medical Center, Cleveland, OH, USA

4 Department of Public Health, Public Health Agency of Catalonia, Lleida, Catalonia, Spain
5 Department of Pathology, University Hospitals Cleveland Medical Center, Cleveland, OH, USA

6 Department of Pathology, Brigham and Women's Hospital, Harvard Medical School, Boston, MA, USA

7 Department of Urology, University Hospitals Cleveland Medical Center, Cleveland, OH, USA

8 Department of Population and Quantitative Health Sciences, School of Medicine, Case Western Reserve University, Cleveland, OH, USA

9 Department of Radiology, University of Michigan, Ann Arbor, MI, USA 